\title{
BRILL-NOETHER THEORY OF SQUAREFREE MODULES SUPPORTED ON A GRAPH
}

\author{
GUNNAR FLØYSTAD AND HENNING LOHNE
}

\begin{abstract}
We investigate the analogy between squarefree CohenMacaulay modules supported on a graph and line bundles on a curve. We prove a Riemann-Roch theorem, we study the Jacobian and gonality of a graph, and we prove Clifford's theorem.
\end{abstract}

\section{INTRODUCTION}

Let $S=k\left[x_{1}, \ldots, x_{n}\right]$ be the polynomial ring. K. Yanagawa ([10] and [11]) introduced the notion of squarefree $\mathbf{Z}^{n}$-graded $S$-modules. Squarefree modules is a generalization of squarefree monomial ideals. These modules are still of a combinatorial nature, as they have support on a simplicial complex. Such modules can be thought of as combinatorial analogues to coherent sheaves, or vector bundles if they are CohenMacaulay.

In this paper we study CM squarefree modules whose support is a graph. These may be thought of as analogues of line bundles on smooth projective curves. Inspired by this we show how results from the theory of line bundles on curves have analogues in the theory of squarefree $\mathrm{CM}$ modules on graphs. We define their degree and global sections, and we show an analog of the Riemann-Roch theorem (Theorem 3.2) for simplical graphs. There is another combinatorial Riemann-Roch theorem for graphs, shown by M. Baker and S. Norine in [2]. Our setting is however different, and our result should be thought of as an algebraic combinatorial analogue. Furthermore, we study squarefree modules of multi-degree $(0, \ldots, 0)$ with support on a graph. This family is an analogue to the Jacobian variety of a curve. We show that the dimension of the family of isomorphism classes of such modules equals the genus of the simplicial graph, similar as for the moduli space of line bundles on a curve.

Then we consider results from Brill-Noether theory, and must then limit ourselves to two-connected graphs. We define effective and special squarefree modules on such graphs, and we define an analogue of

Date: September 20, 2021.

2000 Mathematics Subject Classification. Primary: 13F55, 13C14; Secondary: $14 \mathrm{H} 51$.

Key words and phrases. Cohen-Macaulay modules, Stanley-Reisener rings, Brill-Noether theory. 
gonality. We show that the gonality has the same upper bound as in the classical case. In the end we prove Clifford's theorem for graphs. In Remark 6.10, we briefly discuss the possible connections between these numerical invariants and the resolution of graph curves. A similar study has been done by D. Bayer and D. Eisenbud in [3].

The paper is organized as follows: Section 2 contains preliminaries about squarefree $S$-modules. We also recall a combinatorial description of the canonical module associated to the Stanley-Reisner ring of a simplicial complex.

In Section 3, we describe the analogy between squarefree modules and vector bundles. The main theorem of this section is the analogue of the Riemann-Roch theorem. We also give upper and lower bounds for the degree of an indecomposable squarefree module. We also show the existence of indecomposable squarefree modules of degree $d$ with a one-dimensional space of sections, when $0 \leq d \leq g$, the genus of a graph, analogous the case of line bundles on a curve.

In Section 4, we study squarefree modules of multi-degree $(0, \ldots, 0)$ with support on a simplicial graph. We show that the dimension of the family of isomorphism classes of such modules are the genus of the graph, similar as for the moduli space of line bundles on a curve.

In Section 5, we define effective and special squarefree modules on two-connected graphs, and study the analogue of gonality. The main result is that the gonality has the same upper bound as in the classical case. In the last Section 6 we prove Clifford's theorem

Acknowledgments. The second author thanks Professor Frank-Olaf Schreyer for helpful comments during his stay at the University of Saarland. We are also grateful to the referee for several suggestions to improve the paper, and for pointing out gaps in some of the original proofs.

\section{Preliminaries}

The most natural squarefree modules are squarefree monomial ideals and the corresponding quotient rings. These objects are given by the combinatorial structure of simplicial complexes and Stanley-Reisener rings. We recall the following definitions.

Let $S:=k\left[x_{1}, x_{2}, \ldots, x_{n}\right]$ be the polynomial ring over some field $k$ and let $[n]=\{1,2, \ldots, n\}$. A subset $F \subseteq[n]$ is called a face. A simplicial complex is a collection of faces $\Delta$, such that if $F \in \Delta$ and $G \subseteq$ $F$, then $G \in \Delta$. The Stanley-Reisner ideal of the simplicial complex $\Delta$ is the squarefree monomial ideal $I_{\Delta}=<x^{\sigma} \mid \sigma \notin \Delta>$ generated by monomials corresponding to non-faces $\sigma$ of $\Delta$. The Stanley-Reisner ring of $\Delta$ is the quotient ring $k[\Delta]:=S / I_{\Delta}$

Definition 2.1 (cf. 11] Definition 2.1). A finitely generated $\mathbf{Z}^{n}$-graded $S$-module $M=\bigoplus_{\mathbf{a} \in \mathbf{Z}^{n}} M_{\mathbf{a}}$ is said to be squarefree, if the following hold 
i) $M$ is $\mathbf{N}^{n}$-graded, that is $M_{\mathbf{a}}=0$ for $\mathbf{a} \notin \mathbf{N}^{n}$.

ii) The multiplication map $M_{\mathbf{a}} \stackrel{\cdot x_{i}}{\rightarrow} M_{\mathbf{a}+\mathbf{e}_{i}}$ is bijective for all $\mathbf{a} \in \mathbf{N}^{n}$ and all $i$ in $\operatorname{Supp}(\mathbf{a})$.

Theorem 2.2 (cf. [10] Theorem 2.6). If $M$ is a squarefree $S$-module, then so is $\operatorname{Ext}_{S}^{i}\left(M, \omega_{S}\right)$ for all $i$.

Let $M$ be a $\mathbf{Z}^{n}$-graded $S$-module. The Matlis dual of $M$ is the $\mathbf{Z}^{n}$ graded $S$-module $M^{\vee}=\operatorname{Hom}_{k}(M, k)$. This means that

$$
\left(M^{\vee}\right)_{-\mathbf{a}}=\operatorname{Hom}_{k}\left(M_{\mathbf{a}}, k\right),
$$

and the multiplication map $\left(M^{\vee}\right)_{-\mathbf{a}} \stackrel{\cdot \mathbf{x}^{\mathbf{b}}}{\rightarrow}\left(M^{\vee}\right)_{-\mathbf{a}+\mathbf{b}}$ is the transpose of the multiplication map $M_{\mathbf{a}-\mathbf{b}} \stackrel{\cdot \mathbf{x}^{\mathbf{b}}}{\rightarrow} M_{\mathbf{a}}$. See [7] and [8, Def. 11.15].

Let $H_{m}^{i}(M)$ denote the local cohomology modules of $M$. Then the following holds.

Theorem 2.3 (Local duality). For all finitely generated $\mathbf{Z}^{n}$-graded $\mathbf{S}$ modules $M$ and all integers $i$ there exist natural homogeneous isomorphisms

$$
\left(H_{m}^{i}(M)\right)^{\vee} \cong \operatorname{Ext}_{S}^{n-i}\left(M, \omega_{S}\right)
$$

Proof. See [4, Thm. 3.6.19] or [7, Cor. 6.1].

The local cohomology modules can be calculated by the cocomplex

$$
K^{\bullet}: 0 \rightarrow K^{0} \rightarrow K^{1} \rightarrow \cdots \rightarrow K^{n} \rightarrow 0,
$$

where

$$
K^{i}=\bigoplus_{\substack{\sigma \subseteq[n] \\|\sigma|=i}} M_{\mathbf{x}^{\sigma}}
$$

is the direct sum of the module localized in the squarefree monomials of degree $i$, and the differential is given on each component as

$$
\operatorname{sign}(j, \sigma \cup j) \cdot \text { nat }: M_{\mathbf{x}^{\sigma}} \rightarrow M_{\mathbf{x}^{\sigma \cup j}}
$$

if $j \notin \sigma$, where $\operatorname{sign}(j, \sigma)=(-1)^{\#\{i \in \sigma \mid i<j\}}$. If $M$ is squarefree, then the cohomology $H^{i}\left(K^{\bullet}\right) \cong H_{m}^{i}(M)$ is easy to calculate. As in [10] we have that if $M$ is squarefree, then

$$
\left(M_{\mathbf{x}^{\sigma}}\right)_{-\tau}= \begin{cases}M_{\sigma} & \text { if } \tau \subseteq \sigma \\ 0 & \text { otherwise }\end{cases}
$$

and the natural map $\left(M_{\mathbf{x}^{\sigma}}\right)_{-\tau} \rightarrow\left(M_{\mathbf{x} \sigma \cup\{i\}}\right)_{-\tau}$ corresponds to the map $M_{\sigma} \stackrel{\cdot x_{i}}{\rightarrow} M_{\sigma \cup\{i\}}$.

Definition 2.4. Let $M$ be a squarefree Cohen-Macaulay $S$-module of dimension $d$. The canonical module of $M$ is defined as the squarefree $S$-module

$$
\omega_{M}=\operatorname{Ext}_{S}^{n-d}\left(M, \omega_{S}\right) .
$$


Proposition 2.5. If $M$ is a squarefree Cohen-Macaulay module of dimension $d$ and if $\tau \subseteq[n]$, then

$$
\operatorname{dim}_{k}\left(\omega_{M}\right)_{\tau}=\sum_{\tau \subseteq \sigma \subseteq[n]}(-1)^{d-|\sigma|} \operatorname{dim}_{k} M_{\sigma} .
$$

Proof. If $M$ is Cohen-Macaulay of dimension $d$, then the local cohomology groups $H_{m}^{i}(M)=0$ for $i \neq d$. So the cocomplex $K^{\bullet}$ restricted to degree $-\tau$ has only cohomology $\left(H_{m}^{d}(M)\right)_{-\tau}$, which is isomorphic to $\operatorname{Hom}_{k}\left(\left(\omega_{M}\right)_{\tau}, k\right)$ by local duality. The result now follows since

$$
\left(K^{i}\right)_{-\tau}=\bigoplus_{\substack{|\sigma|=i \\ \tau \subseteq \sigma}} M_{\sigma} .
$$

2.1. The canonical module of $k[\Delta]$. Assume that $\Delta$ is a CohenMacaulay $(d-1)$-dimensional simplicial complex, i.e., the StanleyReisner ring $k[\Delta]$ is Cohen-Macaulay of dimension $d$. The Matlis dual of the cocomplex $K^{\bullet}$ above restricted to positive degrees gives a nice $\mathbf{Z}^{n}$-graded description of $\omega_{k[\Delta]}$. The details are written out in [4, Theorem 5.7.3], here we only give the result.

For each $i=0, \ldots, d$, let $G_{i}$ be the direct sum of the $k[\Delta]$-modules $k\left[X_{1}, \ldots, X_{n}\right] / I_{F}$ where $F \in \Delta,|F|=i$ and $I_{F}=\left(X_{j} \mid j \notin F\right)$. Let $\varphi_{i}: G_{i} \rightarrow G_{i-1}$ be the map which is

$$
(-1)^{j-1} \text { nat }: k\left[X_{1}, \ldots, X_{n}\right] / I_{F} \rightarrow k\left[X_{1}, \ldots, X_{n}\right] / I_{F^{\prime}}
$$

if $F=\left\{v_{i_{1}}, \ldots, v_{i_{r}}\right\}$ and $F^{\prime}=\left\{v_{i_{1}}, \ldots, \widehat{v_{i_{j}}}, \ldots, v_{i_{r}}\right\}$, and zero otherwise. Then the complex

$$
G_{\bullet}: 0 \rightarrow G_{d} \rightarrow G_{d-1} \rightarrow \cdots \rightarrow G_{1} \rightarrow G_{0} \rightarrow 0
$$

is the Matlis dual of $K^{\bullet}$ restricted to positive degrees. When $\Delta$ is Cohen-Macaulay we obtain an exact sequence of $\mathbf{Z}^{n}$-graded $k[\Delta]$-modules

$$
0 \rightarrow \omega_{k[\Delta]} \rightarrow G_{d} \rightarrow G_{d-1} \rightarrow \cdots \rightarrow G_{1} \rightarrow G_{0} \rightarrow 0 .
$$

The description of the canonical module in the long exact sequence above gives rise to a nice description of its squarefree grades. Let st ${ }_{\Delta} F$ denote the set of faces of $\Delta$ containing $F$, and let $\Delta-F$ denote $\Delta \backslash$ st $_{\Delta} F$. We have that

$$
\begin{gathered}
\left(\omega_{k[\Delta]}\right)_{0} \cong \widetilde{H}_{d-1}(\Delta ; k) \text { and } \\
\left(\omega_{k[\Delta]}\right)_{F} \cong \widetilde{H}_{d-1}(\Delta, \Delta-F ; k) \text { for faces } \emptyset \neq F \in \Delta,
\end{gathered}
$$

and the multiplication map

$$
\widetilde{H}_{d-1}(\Delta, \Delta-F ; k) \stackrel{\cdot x_{i}}{\rightarrow} \widetilde{H}_{d-1}(\Delta, \Delta-(F \cup\{i\}) ; k)
$$

is the natural map. 
Remark 2.6. For any face $F$, the homology groups of the chain complex above, restricted to degree $F$, can also be described in the more common way using the link of a face. Recall that the link of $F$ in $\Delta$ is defined as $\operatorname{lk}_{F} \Delta:=\{G \in \Delta \mid F \cap G=\emptyset$ and $F \cup G \in \Delta\}$. The homology groups above can now be described as $\widetilde{H}_{i}(\Delta, \Delta-F ; k) \cong$ $\widetilde{H}_{i-|F|}\left(\mathrm{lk}_{\Delta} F ; k\right)$. This is because the chain complexes are the same. The two different descriptions both have their advantages. The first gives a natural description of the multiplication map, while the second is a combinatorial description.

We use these homology groups to give a characterisation of 2-CM simplicial complexes. Recall that a Cohen-Macaulay simplicial complex $\Delta$ is said to be doubly Cohen-Macaulay or $2-\mathrm{CM}$ if $\Delta-\{p\}$ is Cohen-Macaulay of the same dimension as $\Delta$ for all vertices $p \in \Delta$. The following results might be well-known to specialists. However we give proofs for the reader's convenience.

Proposition 2.7. Let $\Delta$ be a simplicial complex of dimension $d-1$. Then the following are equivalent.

1. $\triangle$ is 2-CM.

2. $\widetilde{H}_{i}(\Delta ; k)=0$ for $0 \leq i \leq d-2$ and $\widetilde{H}_{i}(\Delta-F ; k)=0$ for $0 \leq i \leq d-2$ and any face $F \in \Delta$.

Proof. The homology groups $\widetilde{H}_{i}(\Delta ; k)$ and $\widetilde{H}_{i}(\Delta, \Delta-F ; k)$ are the generators for the module $\operatorname{Ext}_{S}^{n-i-1}\left(k[\Delta], \omega_{S}\right)$ by the complex (2), and by local duality Theorem 2.3 . Because of the long exact sequence

$$
\begin{aligned}
\cdots & \rightarrow \widetilde{H}_{i}(\Delta ; k) \rightarrow \widetilde{H}_{i}(\Delta, \Delta-F ; k) \rightarrow \widetilde{H}_{i-1}(\Delta-F ; k) \\
& \rightarrow \widetilde{H}_{i-1}(\Delta ; k) \rightarrow \cdots
\end{aligned}
$$

we see that $\Delta$ is $\mathrm{CM}$ if and only if $\widetilde{H}_{i}(\Delta ; k)=0$ for $0 \leq i \leq d-2$ and $\widetilde{H}_{i}(\Delta-F ; k)=0$ for $0 \leq i \leq d-3$ and all faces $\emptyset \neq F \in \Delta$. So it is enough to show that $\Delta$ is 2-CM if and only if $\Delta$ is $\mathrm{CM}$ and that $\widetilde{H}_{d-2}(\Delta-F ; k)=0$ for any face $F \in \Delta$.

Suppose that $\Delta$ is 2 -CM. We will show that $\widetilde{H}_{d-2}(\Delta-F ; k)=0$ for all faces $F \in \Delta$ using induction on the dimension of $F$. If $F=\{p\}$ is just a vertex, then $\widetilde{H}_{d-2}(\Delta-\{p\} ; k)=0$ since $\Delta-p$ is CM. Now suppose that $G=F \cup\{p\}$ is a face. Let $A=\Delta-F$ and $B=\Delta-\{p\}$. Then $A \cup B=\Delta-G$ and $A \cap B=(\Delta-\{p\})-F$. We therefore have a Mayer-Vietoris sequence

$$
\begin{aligned}
\cdots & \rightarrow \widetilde{H}_{d-2}(\Delta-F ; k) \oplus \widetilde{H}_{d-2}(\Delta-\{p\} ; k) \rightarrow \widetilde{H}_{d-2}(\Delta-G ; k) \\
& \rightarrow \widetilde{H}_{d-3}((\Delta-\{p\})-F ; k) \rightarrow \cdots .
\end{aligned}
$$


The homology groups on the left side are trivial because of the induction hypothesis and the homology group on the right side is trivial because $\Delta-\{p\}$ is $\mathrm{CM}$ of dimension $d-1$ so it follows that $\widetilde{H}_{d-2}(\Delta-G ; k)=0$.

Suppose that $\Delta$ is $\mathrm{CM}$ and that $\widetilde{H}_{d-2}(\Delta-F ; k)=0$ for all faces $F \in \Delta$. We have that $\widetilde{H}_{i}(\Delta-\{p\} ; k)=0$ for $0 \leq i \leq d-2$. Let $p \in \Delta$ be a vertex and $F \in \Delta$ a face not containing $p$. As above, we get a Mayer-Vietoris sequence

$$
\begin{aligned}
\cdots \rightarrow \widetilde{H}_{i+1}(\Delta-G ; k) & \rightarrow \widetilde{H}_{i}((\Delta-\{p\})-F ; k) \\
& \rightarrow \widetilde{H}_{i}(\Delta-\{p\} ; k) \oplus \widetilde{H}_{i}(\Delta-F ; k) \rightarrow \cdots,
\end{aligned}
$$

where $G$ is the face $F \cup\{p\}$ if this is a face in $\Delta$, otherwise, we just replace $\Delta-G$ above with $\Delta$. For $0 \leq i \leq d-3$, the homology group on the left side is trivial because of the assumption and the fact that $\Delta$ is $\mathrm{CM}$, and the homology groups on the right side are trivial because $\Delta$ is $\mathrm{CM}$, so $\widetilde{H}_{i}((\Delta-\{p\})-F ; k)=0$ for $0 \leq i \leq d-3$ and it follows that $\Delta-p$ is $\mathrm{CM}$ for all vertices $p \in \Delta$, hence $\Delta$ is 2-CM.

This characterisation of 2-CM simplicial complexes has the following corollary, which we will use later. For more details see [1], 44] and [9].

Corollary 2.8. Let $\Delta$ be a CM simplicial complex of dimension $d-$ 1. Then $\Delta$ is $2-C M$ if and only if $\omega_{k[\Delta]}$ is generated by $\left(\omega_{k[\Delta]}\right)_{0} \cong$ $\widetilde{H}_{d-1}(\Delta ; k)$.

Proof. Consider the long exact sequence

$0 \rightarrow \widetilde{H}_{d-1}(\Delta-F) \rightarrow \widetilde{H}_{d-1}(\Delta) \stackrel{\cdot \mathbf{x}^{F}}{\rightarrow} \widetilde{H}_{d-1}(\Delta, \Delta-F) \rightarrow \widetilde{H}_{d-2}(\Delta-F) \rightarrow 0$.

$\omega_{k[\Delta]}$ is generated by $\widetilde{H}_{d-1}(\Delta ; k)$ if and only if $\cdot \mathbf{x}^{F}$ is surjective for every $F$. That is, if and only if $\widetilde{H}_{d-2}(\Delta-F)=0$ for every face $F$.

\section{The Riemann-Roch TheOREM}

The annihilator of a squarefree $S$-module $M$ is a squarefree monomial ideal. Since squarefree monomial ideals corresponds to simplicial complexes, $M$ can be considered as a module over the Stanley-Reisner ring $k[\Delta]=k\left[x_{1}, \ldots, x_{n}\right] / \operatorname{ann}(M)$. The study of squarefree CohenMacaulay modules are therefore the same as the study of maximal Cohen-Macaulay modules over Stanley-Reisner rings $k[\Delta]$, with support on all of $k[\Delta]$. A squarefree $S$-module can be described as follows. For each face $A \subseteq[n]$ we have a $k$-vector space $M_{A}$, and for each face $A \subseteq B$ we have a $k$-linear map $\varphi_{A B}: M_{A} \rightarrow M_{B}$ satisfying $\varphi_{A A}=i d_{M_{A}}$ and if $A \subseteq B \subseteq C$, then $\varphi_{B C} \circ \varphi_{A B}=\varphi_{A C}$.

In the rest of this paper we study squarefree Cohen-Macaulay modules with support on a graph. We always assume the graph to be 
connected, simple and without isolated vertices, so it is a CM onedimensional simplicial complex. First note the following simple criterion.

Lemma 3.1. A squarefree module $M$ with support a graph is $C M$ if and only if (the $e$ denote edges):

1. For each vertex $v$ the map

$$
M_{v} \stackrel{\oplus \phi_{v e}}{\longrightarrow} \oplus_{v \in e} M_{e}
$$

is injective.

2. The following sequence is left exact:

$$
0 \rightarrow M_{\emptyset} \stackrel{\oplus \phi_{\emptyset v}}{\longrightarrow} \underset{\text { vertices } v}{\oplus} M_{v} \stackrel{\oplus \phi_{\emptyset v}}{\longrightarrow} \underset{\text { edges e }}{\oplus} M_{e} .
$$

Proof. That $M$ is CM means that the complex (11) has cokernel only in cohomological degree 2 . When considering it in degree $\{v\}$ we get 1 . above, and when considering it in degree $\emptyset$ we get 2 .

Such a module $M$ on a graph $G$ gives rise to a sheaf $\widetilde{M}$ on Proj $k[G]$ and there is a natural graded isomorphism between $M$ and the graded module of global sections $\bigoplus_{\tau} \Gamma(\operatorname{Proj} k[G], \widetilde{M}(\tau))$ [6, Appendix A4]. We shall consider such modules with $M_{e}$ one-dimensional for all edges $e$ in the graph. In this case we say that $M$ is locally of rank 1 . Such a module may be seen as the analog of a line bundle on a curve.

Inspired by this, we define $l(M):=\operatorname{dim}_{k} M_{\emptyset}$ as an analogue of the global sections. Furthermore, we define the multi-degree of $M$ as the vector $\mathbf{d} \in \mathbf{Z}^{n}$, such that $d_{i}=\operatorname{dim}_{k} M_{\{i\}}-1$, and the degree of $M$ as $\operatorname{deg}(M):=\sum_{i} d_{i}$. This definition of the degree of a module is an analogy to the degree of a line bundle.

Let $V$ be the vertices of the graph $G$ and $E$ its set of its edges. We define the genus of $G$ as:

$$
g(G):=l\left(\omega_{k[G]}\right)=\operatorname{dim}_{k}\left(\omega_{k[G]}\right)_{0}=\operatorname{dim}_{k} \widetilde{H}_{1}(G ; k)=1-|V|+|E| .
$$

Theorem 3.2 (Riemann-Roch). Let $G$ be a graph, and $M$ a squarefree Cohen-Macaulay module with support on $G$, locally of rank 1 . Then the following formula holds.

$$
l(M)-l\left(\omega_{M}\right)=1+\operatorname{deg}(M)-g .
$$

Proof. Using Proposition 2.5, we can calculate the left hand side as

$$
\sum_{|\sigma|=1} M_{\sigma}-\sum_{|\tau|=2} M_{\tau}=(\operatorname{deg}(M)+|V|)-|E|=\operatorname{deg}(M)+1-g .
$$

Using the Riemann-Roch formula, it is easy to see that $\operatorname{deg}\left(\omega_{M}\right)=$ $2 g-2-\operatorname{deg}(M)$. Since the degree is obviously bounded below by $\operatorname{deg}(M) \geq-|V|$ and $\omega_{M}$ is a squarefree Cohen-Macaulay module 
whenever $M$ is, we get that the degree is also bounded above by $\operatorname{deg}(M) \leq 2 g-2+|V|$. For a given graph, it is possible to give a better bound for the degree.

If $\tau$ is a subset of the vertices, the restriction $\left.G\right|_{\tau}$ is the graph whose vertices are $\tau$ and whose edges are the edges in $G$ with both endpoints in $\tau$.

Proposition 3.3. Let $G$ be a graph, and $M$ an indecomposable $C M$ squarefree module on $G$, locally of rank 1 . Then

$$
-s \leq \operatorname{deg}(M) \leq 2 g-2+s,
$$

where

$$
s=\max \left\{|\sigma| \mid \sigma \subseteq V, \operatorname{dim}\left(\left.G\right|_{\sigma}\right)=0 \text { and }\left.G\right|_{\sigma^{c}} \text { is connected }\right\} .
$$

Furthermore, for any $-s \leq i \leq 2 g-2+s$ there exists such a module $M$ with $\operatorname{deg}(M)=i$.

Proof. Since the upper bound is the dual of the lower bound, it is enough to show that the lower bound holds. Suppose that $\operatorname{deg}(M)=$ $-k$. Then there are at least $k$ vertices in $G$ where $M_{v}=0$. Let $\tau \subseteq[n]$ be the subset that corresponds to these vertices. Since $M$ is indecomposable, $\left.G\right|_{\tau^{c}}$ must be connected and there can not be any edges in $\left.G\right|_{\tau}$, so $\operatorname{dim}\left(\left.G\right|_{\tau}\right)=0$. It therefore follows from the definition of $s$ in the proposition that $-s \leq-k$.

For $0 \leq i \leq s$, let $\sigma \subseteq[n]$ be a subset such that $|\sigma|=i, \operatorname{dim}\left(\left.G\right|_{\sigma}\right)=$ 0 and $\left.G\right|_{\sigma^{c}}$ is connected. Then we can construct an indecomposable module $M$ with $\operatorname{deg}(M)=-i$. Let $M$ be the module where $M_{v}=0$ for all vertices $v \in \sigma, M_{v}=k 1_{v}$ for all vertices $v \in \sigma^{c}$ and $M_{e}=k 1_{e}$ for all edges, with multiplication map $\varphi_{v e}\left(1_{v}\right)=1_{e}$ for all vertices $v \in \sigma^{c}$ and edges $e$ containing $v$.

If $M$ is decomposable, isomorphic to $M^{\prime} \oplus M^{\prime \prime}$, let $V^{\prime}$ be the vertices in $\sigma^{c}$ at which $M_{v}^{\prime}$ is nonzero and $V^{\prime \prime}$ the vertices of $\sigma^{c}$ at which $M^{\prime \prime}$ is nonzero. Then $V^{\prime} \cup V^{\prime \prime}$ is a partition of $\sigma^{c}$. The edges of $G$ may be partitioned into $E^{\prime} \cup E^{\prime \prime}$ where $E^{\prime}$ are the edges in the support of $M^{\prime}$ and correspondingly for $E^{\prime \prime}$. Note that since the maps $\phi_{v e}$ are all nonzero when $v \in \sigma^{c}$ and $v \in e$, an edge in $E^{\prime}$ must have both endpoints in $V^{\prime}$. Similarly for $E^{\prime \prime}$. But this implies that $\left.G\right|_{\sigma} ^{c}$ is disconnected, contrary to assumption. So $M$ is indecomposable of degree $-i$. By duality it also follows that we can construct the dual module, which has degree $2 g-2+i$.

It remains to show that there exist modules with degree in the range $0 \leq \operatorname{deg}(M) \leq 2 g-2$. Again by duality, it is enough to show the existence of modules with $0 \leq \operatorname{deg}(M) \leq g$. Such modules are interesting, and their existence will be showed in the proposition below.

The following result, which is also an analogue of a well-known fact from algebraic geometry, is needed to complete the proof of the previous proposition. 
Proposition 3.4. Let $G$ be a graph of genus $g$. For any $0 \leq d \leq g$, there exists an indecomposable $C M$ module $M$ with support $G$, locally of rank 1 , of degree $d$, multi-degree $\geq(0,0, \ldots, 0)$, and with a onedimensional space of global sections, i.e. $l(M)=1$.

Proof. If $d=0$ we can choose $M=k[G]$. Otherwise $0<d \leq g$. Let $e_{0}=\left\{v_{0}, w_{0}\right\}$ be en edge of $G$ whose removal gives a subgraph $H$ of genus $g-1$. By induction we may assume there is a module $N$ on $H$ of degree $d-1$ and with the other properties stated in the proposition. Make the module $M$ on $G$ such that $M_{\sigma}=N_{\sigma}$ when $\sigma \neq v_{0}, e_{0}$, but $M_{e_{0}}=k \cdot 1_{e_{0}}$, and $M_{v_{0}}=N_{v_{0}} \oplus k \cdot 1_{v_{0}}$. Define the $k$-linear maps $\varphi_{v e}$ of $M$ to be general maps extending those of $N$, subject to the commutativity constraint, i.e. they give an $S$-module structure on $M$. The degree of $M$ is $d$ and we see by Lemma 3.1 that $M$ will be a CM module with $l(M)=l(N)=1$.

\section{THE JACOBIAN}

We study the moduli space of isomorphism classes of squarefree CM $S$-modules, with support on a graph, of multi-degree $(0,0, \ldots, 0)$. This space is not as nice as the Jacobi variety of an algebraic curve. However, if we restrict to "non-degenerate" modules we can give the space an algebraic structure with dimension equal to the genus of the graph.

Lemma 4.1. Let $G$ be a tree, and $M$ a squarefree indecomposable module (possible not $C M$ ) of multi-degree $(0,0, \ldots, 0)$ with support $G$, locally of rank 1.

For the vertices $v$ we may then choose generators $1_{v}$ of $M_{v}$ and similarly $1_{e}$ for the edges e such that whenever $v \in e$ then $\varphi_{v e}$ sends $1_{v}$ to $1_{e}$. In particular there is only one such CM module up to isomorphism, and it will have a one-dimensional space of global sections.

Proof. We do induction on the number of vertices on $G$. Let $v$ be a leaf of $G$, i.e. a vertex of degree one, and $e=v w$ the incident edge. Removing $v$ and $e$ we get a tree $H$. By induction it is enough to show that $\varphi_{w e}\left(1_{w}\right)=1_{e}$ and $\varphi_{v e}\left(1_{v}\right)=1_{e}$. However, since both $\varphi_{w e}$ and $\varphi_{v e}$ are non-zero (if one of them is zero then $M$ is decomposable) we can first find a basis of $M_{e}$ such that $\varphi_{w e}=1$, and next find a basis for $M_{v}$ such that $\varphi_{v e}=1$. The same technique can be used to show the case where $G$ has only one edge.

That $M$ has a one-dimensional space of global sections when $M$ is CM follows from Lemma 3.1.

Proposition 4.2. Let $G$ be a cycle. The family of indecomposable $C M$ modules over $k[G]$, locally of rank 1 , with multi-degree $(0,0, \ldots, 0)$ is parametrized by a union of $n \mathbb{P}_{k}^{1}$ 's, where we identify the same points from the different $\mathbb{P}_{k}^{1}$ 's if they are not $(0,1)$ or $(1,0)$. In other words, it is parametrized by a $\mathbb{P}_{k}^{1}$, with the points $(0,1)$ and $(1,0)$ n-doubled. 
Proof. Suppose all the $\varphi_{v e}$ are nonzero when $v \in e$. Let $e$ be an edge of $G$ and $H$ the line graph obtained by removing $e$ from $G$. Then $\left.M\right|_{H}$ has the $k$-linear maps as described in the lemma above. $M$ is therefore determined by the two $k$-linear maps $\varphi_{v e}$ and $\varphi_{w e}$, where $e=v w$. For any basis of $M_{e}$, these maps are determined by a pair $(s, t) \in k^{2}$. Since any basis change of $M_{e}$ is multiplication by a non-zero element of $k$, it follows that the maps are determined, up to isomorphism, by an element $(s, t)$ in $\mathbb{P}_{k}^{1} \backslash\{(1,0),(0,1)\}$. Note that the latter is isomorphic to $\mathbb{A}_{k}^{1} \backslash\{0\}$.

If two distinct $\varphi_{v e}$ are zero with $v$ incident to $e$ then $M$ is decomposable. So assume exactly one such $\varphi_{v_{0} e_{0}}$ is zero. Then as in the case that $G$ is a tree that we may find generators $1_{v}$ for the $M_{v}$ and $1_{e}$ for the $M_{e}$ such that all other $\varphi_{v e}$ sends $1_{v}$ to $1_{e}$ whenever $v \in e$. Hence for each $v_{0} \in e_{0}$ there is exactly one isomorphism class of such modules. If we have a cyclic order on the vertices, these $2 n$ pairs may be identified with $n$ copies of $(1,0)$ and $n$ copies of $(0,1)$.

In the cases of the first paragraph we will by Lemma 3.1 get CM modules with no global sections if $(s, t) \neq(1,1)$ in $\mathbb{P}^{1}$ and with a onedimension space of global sections, i.e. $l(M)=1$ if $(s, t)=(1,1)$. In the cases of the second paragraph we get no global sections.

Proposition 4.3. Let $G$ be a graph of genus $g \geq 1$. The family of indecomposable $C M$ modules over $k[G]$, locally of rank 1 , with multidegree $(0,0, \ldots, 0)$ and where all the maps $\varphi_{v e}$ are non-zero when $v \in e$, is parametrized by $\left(\mathbb{A}_{k}^{1} \backslash\{0\}\right)^{g}$.

Proof. We may choose a set $E^{\prime}$ of $g$ edges such that $\left(V, E \backslash E^{\prime}\right)$ is a tree. Given a module $M$ on $G$ of multidegree $(0, \ldots, 0)$ we may by Lemma 4.1 for the vertices $v$ choose generators $1_{v}$ of $M_{v}$ and similarly generators $1_{e}$ for $M_{e}$ for the edges in $E \backslash E^{\prime}$ such that $\phi_{v e}\left(1_{v}\right)=1_{e}$. This choice of generators is unique up to multiplication by a common scalar.

The module $M$ is now specified by for each edge $e=v w$ in $E^{\prime}$ choosing a pair of maps $\varphi_{v e}$ and $\varphi_{w e}$. For a choice of generator $1_{e}$ of $M_{e}$ a pair of maps is given by a pair $(s, t)$ where $s, t \neq 0$. We may change the generator for $M_{e}$ by multiplying by a scalar, or change the set of generators $\left\{1_{v}\right\}$ by multiplying by a common scalar. This shows that such $M$ are parametrized by $\left(\mathbb{P}_{k}^{1} \backslash\{(1,0),(0,1)\}\right)^{g}$ which is isomorphic to $\left(\mathbb{A}_{k}^{1} \backslash\{0\}\right)$.

\section{Gonality}

As we have seen, there is an analogy between line bundles on a given curve and squarefree Cohen-Macaulay modules with support on a given graph. We investigate this further by defining effective and special 
modules as an analogue of effective special divisors. The corresponding "Brill-Noether" theory for squarefree Cohen-Macaulay modules has some similarities and some differences from the classical theory.

Definition 5.1. An indecomposable squarefree CM module $M$ supported on a graph $G$ is said to be effective if $M$ has a submodule isomorphic to $k[G]$, and said to be special if $M \subseteq \omega_{k[G]}$.

A graph is CM if and only if it is connected, and 2-CM if and only if it is 2-connected. From Corollary 2.8, we know that the canonical module $\omega_{k[G]}$ is generated by the cycles $\widetilde{H}_{1}(G ; k)$ if and only if $G$ is 2-connected. It is therefore natural to study special modules of 2-connected graphs.

Some of the following results are using that the field $k$ is infinite. We are therefore assuming that $\operatorname{char}(k)=0$ for the rest of this section. Recall that the cycles $Z_{1}(G)$ is the kernel of the boundary map from the one-chains to the zero-chains

$$
C_{1}=k E \rightarrow k V=C_{0}
$$

Lemma 5.2. Let $G$ be a two-connected graph. Then $\omega_{k[G]}$ has a submodule $N \cong k[G]$ such that any squarefree module $N \subseteq M \subseteq \omega_{k[G]}$ is indecomposable, and therefore effective.

Proof. Let $u_{N}$ be a general element in $Z_{1}(G)$. Then $\operatorname{Supp}\left(u_{N}\right)=G$. Let $N$ be the submodule of $\omega_{k[G]}$ generated by $u_{N}$ and suppose that $M$ is a module $N \subseteq M \subseteq \omega_{k[G]}$. Suppose that $M=M^{\prime} \oplus M^{\prime \prime}$ is decomposable. Let $V^{\prime}$ be the vector space spanned by the elements of $M^{\prime}$ in degree 0 , and $V^{\prime \prime}$ the vector space spanned by the corresponding elements of $M^{\prime \prime}$. We will show that there exists a cycle $s \in Z_{1}(G)$ such that $s \notin V^{\prime} \oplus V^{\prime \prime}$. We construct $s$ as follows: Let $G^{\prime}:=\operatorname{Supp} M^{\prime}$ and $G^{\prime \prime}:=\operatorname{Supp} M^{\prime \prime}$. We must have that $\operatorname{dim} G^{\prime} \cap G^{\prime \prime}<1$. This is because $M_{e} \cong k$ for every edge $e$ in $G$, which means that each edge of $G$ must either be in $G^{\prime}$ or in $G^{\prime \prime}$. Since $G$ is connected, there has to be at least one vertex in this intersection. Let $v$ be any such vertex, and let $v^{\prime}$ be a vertex adjacent to $v$ in $G^{\prime}$ and $v^{\prime \prime}$ a vertex adjacent to $v$ in $G^{\prime \prime}$. Furthermore, since $G$ is two-connected, $G-\{v\}$ is connected and there is a simple path from $v^{\prime}$ to $v^{\prime \prime}$. Let $s \in Z_{1}(G)$ be a cycle corresponding to the simplicial circle obtained by connecting this path in $v$. This cycle is clearly not in $V^{\prime} \oplus V^{\prime \prime}$, since $s$ cannot be written as a sum of two cycles with disjoint support. This means that $\operatorname{dim}_{k} V^{\prime} \oplus V^{\prime \prime}<\operatorname{dim}_{k} Z_{1}(G)$. Any decomposition of a module $M$ like this corresponds to a partitioning $E=E^{\prime} \cup E^{\prime \prime}$ of the edges in $G$, and in all cases, we will have as above that $\operatorname{dim}_{k} V^{\prime} \oplus V^{\prime \prime}<\operatorname{dim}_{k} Z_{1}(G)$. Since there are only finitely many ways to partitioning the edges into two sets, we can only find a decomposable $M$ between $N$ and $\omega_{k[G]}$ if $u_{N}$ lies in this finite union of subspaces of codimension $\geq 1$. But since $u_{N}$ is a general element, this is not the case. 
Definition 5.3. Let $G$ be a 2-connected graph of genus $g \geq 2$. A special and effective module $M$ satisfying $l(M) \geq r+1$ and $\operatorname{deg}(M)=d$ is called a $g_{d}^{r}$. We define the gonality of a graph to be

$$
\operatorname{gon}(G)=\min \left\{k \mid G \text { possesses a } g_{k}^{1}\right\} \text {. }
$$

For curves, it is well known that the gonality lies between 2 and $\left\lfloor\frac{g+3}{2}\right\rfloor$. As we will see below, the same holds for a two-connected graph.

Lemma 5.4. Let $G$ be a two-connected graph of genus $g \geq 2$. Then the gonality of $G$ is less then or equal to the girth (i.e. the length of the shortest nonzero cycle) of $G$.

Proof. From Lemma [5.2, we can choose $N \subseteq \omega_{k[G]}$ such that $N \cong k[G]$. Choose the cycle $u_{N} \in N$ and a cycle $c$ of length $w=\operatorname{girth}(G)$. Then the submodule $M \subseteq \omega_{k[G]}$ generated by $u_{N}$ and $c$ is a $g_{d}^{1}$, where $d \leq w$ since $\operatorname{Supp}\left(u_{N}\right) \cap \operatorname{Supp}(c)$ contains no more than $w$ vertices.

Lemma 5.5. Let $G$ be a two-connected graph of genus $g \geq 3$ and minimum valency $k \geq 3$. Then the girth of $G$ is $\leq\left\lfloor\frac{g+3}{2}\right\rfloor$ except for three special cases:

- $K_{3,3}$,

- The Petersen graph,

- The Heawood graph.

Proof. According to [5], Theorem 11.11.3, we have that the number of vertices of $G$, denoted by $v$, is bounded by

$$
2 g-2 \geq v \geq 1+k \frac{(k-1)^{\left\lfloor\frac{w-1}{2}\right\rfloor}-1}{k-2} .
$$

Suppose that the girth $w \geq\left\lfloor\frac{g+5}{2}\right\rfloor$, then the inequality above has only finitely many solutions. The possible solutions are given as follows:

\begin{tabular}{|c|c|c|}
\hline$g$ & $v$ & $w$ \\
\hline 3 & 4 & 4 \\
\hline 4 & $4-6$ & 4 \\
\hline 6 & 10 & 5 \\
\hline 7 & $10-12$ & 6 \\
\hline 8 & $10-14$ & 6 \\
\hline 12 & 22 & 8 \\
\hline
\end{tabular}

However, the lower bound can be sharpened. If $w=4$ then $G$ must have a subgraph as in the picture below, as we now explain.

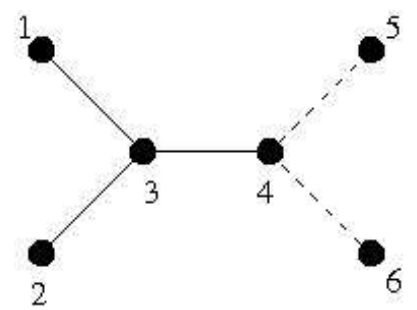


Since $G$ has minimum valency $k \geq 3$, it is clear that it has a subgraph consisting of vertices 1-4. But furthermore, since the vertex 4 has valency $\geq 3$, and since the girth is 4 there must exist two other vertices adjacent to 4 . Likewise, if $w=6$ and $G$ has minimum valency $k \geq 3$ then $G$ must contain a subgraph like the following figure.

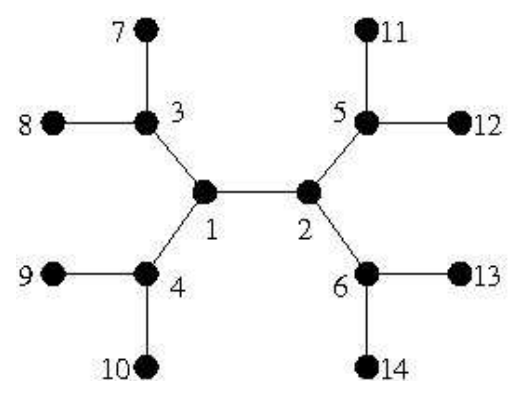

The reason for this is the following. $G$ must contain a vertex, so 1 is in $G$. Since $G$ has minimum valency $k \geq 3$, there has to be three vertices adjacent to 1 , call them 2,3 and 4 . The vertex 2 must also be adjacent to three vertices. But since the girth $w=6$ it is not adjacent to the vertex 2 or 3 . Therefore it must be adjacent to two new vertices, call them 5 and 6 . The same logic applies to the vertices 3,4,5 and 6 , and it is clear that $G$ contains a subgraph as in the figure.

For $w=8, G$ must also contain a subgraph as above. However, it is not so difficult to see, using the same argument as above, that it is impossible to construct a graph with $w=8$ and $k \geq 3$ with only 8 more vertices. Therefore most of the solutions from the inequality above can not correspond to a graph. The only graphs satisfying the inequality in the Lemma that can exist have the following numerical data.

\begin{tabular}{|c|c|c|}
\hline$g$ & $v$ & $w$ \\
\hline 4 & 6 & 4 \\
\hline 6 & 10 & 5 \\
\hline 8 & 14 & 6 \\
\hline
\end{tabular}

There is exactly one graph for each of the cases above. The first is $K_{3,3}$, the second is the Petersen graph and the last is the Heawood graph.

For any two-connected graph we would like to find a "canonical" model for it. Let $G$ be a two-connected graph of genus $g \geq 2$. If $v \in G$ is a vertex of valency 2 and $e_{1}=v w_{1}$ and $e_{2}=v w_{2}$ are the edges that meet $v$ and $e^{\prime}=w_{1} w_{2}$ is not an edge in $G$. Then we construct a new graph $G^{\prime}=(G-v) \cup\left\{e^{\prime}=w_{1} w_{2}\right\}$. If we iterate this process, we end up with a graph we call $\widetilde{G}$. It is easy to verify that $\widetilde{G}$ has the same topological structure as $G$. We will say that a graph $G$ is reduced if $G=\widetilde{G}$. 
Lemma 5.6. Let $G$ be a two-connected graph of genus $g \geq 2$. Then $\operatorname{gon}(G)=\operatorname{gon}(\widetilde{G})$, and $\widetilde{G}$ is either hyperelliptic, i.e. 2-gonal, or has minimal valency $k \geq 3$.

Proof. Let $M$ be an effective module on $G$, and let $v$ be a vertex as described above. Then $k[G]_{v} \subseteq M_{v} \subseteq\left(\omega_{k[G]}\right)_{v}$ but $k[G]_{v} \cong k \cong\left(\omega_{k[G]}\right)_{v}$ since $v$ has valency 2 . This means that $M_{v} \cong k$, and it does not contribute to the degree of $M$. That means that for any effective module on $G$ we can construct an effective module $M^{\prime}$ on $G^{\prime}$ such that $M^{\prime}$ has the same numerical invariants as $M$. Iterating this process we get an effective module $\widetilde{M}$ on $\widetilde{G}$ with the same numerical invariants as $M$. It is also easy to see that any effective module $N$ on $\widetilde{G}$ can be obtained from an effective module coming from $G$. This proves the first part.

For the other part, we notice that either $\widetilde{G}$ has minimal valency $k \geq 3$ or there exists a vertex $v$ of valency 2 such that if $e_{1}=v w_{1}$ and $e_{2}=v w_{2}$ are the edges that meet $v$, then $e^{\prime}=w_{1} w_{2}$ is an edge in $\widetilde{G}$. That means that there exists a cycle $s \in Z^{1}(G)$ such that $\operatorname{Supp}(s)=$ $e_{1} \cup e_{2} \cup e^{\prime}$. Since $G$ is 2-connected we can find a submodule $N \subseteq \omega_{k[G]}$, isomorphic to $k[G]$, such that any module in between is effective. Let $u_{N}$ be the generator of $N$, then $M=\left(u_{N}, s\right)$ is a $g_{d}^{1}$ where $d \leq 3$ since $\operatorname{Supp}\left(u_{N}\right) \cap \operatorname{Supp}(s)$ contains 3 vertices. Furthermore, $d \leq 2$ since $v$ has valency 2 , and $d \geq 2$ sine $G$ is two-connected. Hence $\widetilde{G}$ has gonality 2 , that is $\widetilde{G}$ is hyperelliptic.

Theorem 5.7. Let $G$ be a two-connected graph of genus $g \geq 2$. Then $2 \leq \operatorname{gon}(G) \leq\left\lfloor\frac{g+3}{2}\right\rfloor$.

Proof. That the gonality cannot be 1 is clear since $G$ is two-connected. By the previous two Lemmata, it is enough to show that there exists a $g_{\left\lfloor\frac{g+3}{2}\right\rfloor}^{1}$ on the three special graphs above. The graph $K_{3,3}$ can be drawn like this:

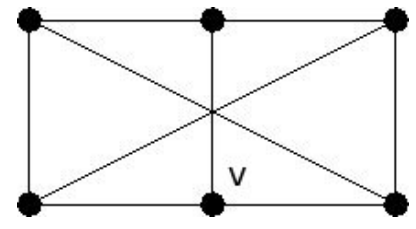

We notice the two subgraphs

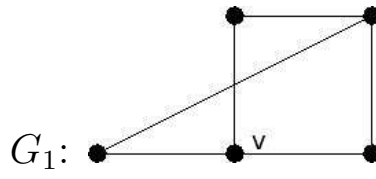

and

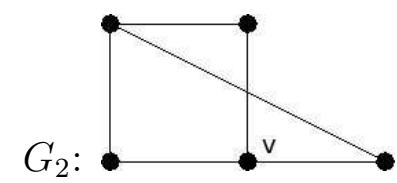

Let $s_{1} \in Z^{1}(G)$ be a cycle with support on $G_{1}$. We claim that there exists a cycle $s_{2} \in Z^{1}(G)$ with support on $G_{2}$ such that the module $M$ generated by $s_{1}$ and $s_{2}$ is a $g_{3}^{1}$. First of all we notice that for any $s_{2}$ with support on $G_{2}$ we have that $M$ is effective since $\operatorname{Supp}\left(s_{1}, s_{2}\right)=G$, 
and indecomposable since $\operatorname{dim} G_{1} \cap G_{2}=1$. Next, we show that we can choose $s_{2}$ such that the restrictions of $s_{1}$ and $s_{2}$ are equal in the vertex $v$. This follows if we show that we can choose $s_{2}$ such that the restrictions of $s_{1}$ and $s_{2}$ are equal in the three edges of $v$, and this is so since the two four-cycles of $G_{2}$ are linearly independent at the vertex $v$. We now have

$$
\operatorname{deg}(M)=\sum_{\text {vertices } v}\left(\operatorname{dim}_{k}\left(\operatorname{Span}\left\{\left.s_{1}\right|_{v},\left.s_{2}\right|_{v}\right\}\right)-1\right)=3 .
$$

The Petersen graph is given as:

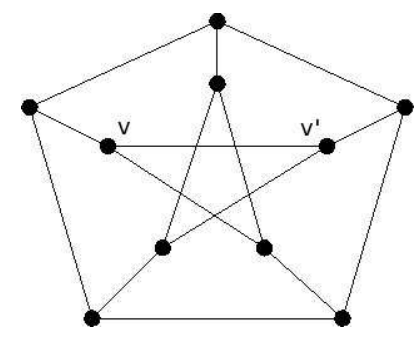

We notice the two subgraphs

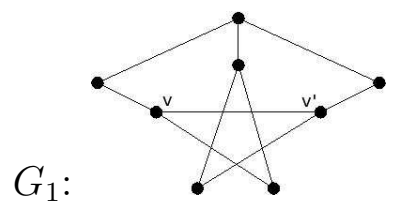

and

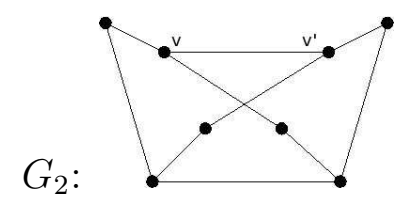

Let $s_{1}$ be a cycle with support on $G_{1}$. As above, we claim that there exists a cycle $s_{2}$ with support on $G_{2}$ such that the module $M$ generated by $s_{1}$ and $s_{2}$ is a $g_{4}^{1}$. For any $s_{2}$ with support on $G_{2}$ we have that $M$ is effective and indecomposable for the same reasons as above. Next, we show that we can choose $s_{2}$ such that the restrictions of $s_{1}$ and $s_{2}$ are equal in the vertices $v$ and $v^{\prime}$. As above, this follows if we can choose $s_{2}$ such that the restrictions of $s_{1}$ and $s_{2}$ are equal on any two of the edges of $v$ and any two of the edges of $v^{\prime}$. Again, this follows since $G_{2}$ has three linearly independent cycles, $v$ and $v^{\prime}$ each has valency 3 , and cycles are independent in these vertices. Similarly as above, we now get that $\operatorname{deg}(M)=4$.

The Heawood graph is given as:

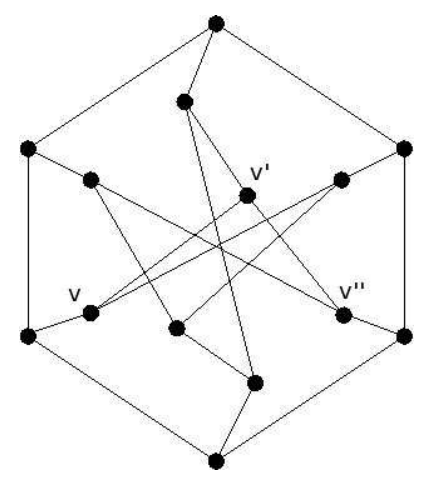


We notice the two subgraphs

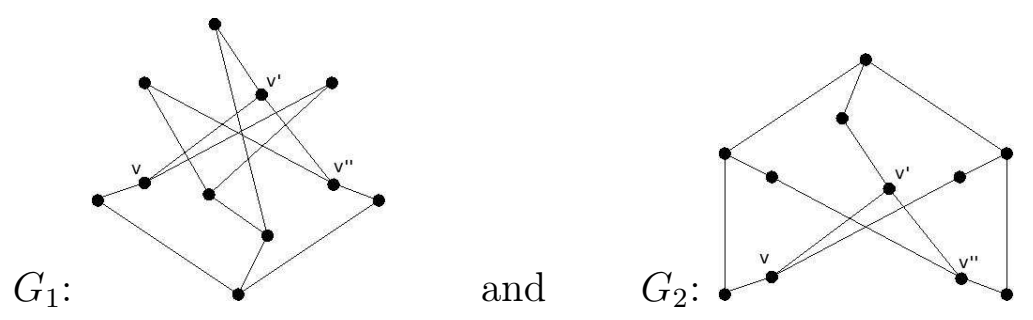

Let $s_{1} \in \widetilde{H}_{1}(G ; k)$ be a cycle with support on $G_{1}$. As above, we claim that there exists a cycle $s_{2} \in \widetilde{H}_{1}(G ; k)$ with support on $G_{2}$ such that the module $M$ generated by $s_{1}$ and $s_{2}$ is a $g_{5}^{1}$. For any $s_{2}$ with support on $G_{2}$ we have that $M$ is effective and indecomposable for the same reasons as above. Next, we show that we can choose $s_{2}$ such that the restrictions of $s_{1}$ and $s_{2}$ are equal in the vertices $v, v^{\prime}$ and $v^{\prime \prime}$. As above, this follows if we can choose $s_{2}$ such that the restrictions of $s_{1}$ and $s_{2}$ are equal on any two of the edges of $v, v^{\prime}$ and $v^{\prime \prime}$. Again, this follows since $G_{2}$ has four linearly independent cycles and the three vertices $v, v^{\prime}$, and $v^{\prime \prime}$ each have valency 3 . Similarly as above, we now get that $\operatorname{deg}(M)=5$.

One may ask which gonalities that occur in the bound. It is wellknown that in the moduli space of curves of genus $g$, there exist curves of gonality $k$ for all $2 \leq k \leq\left\lfloor\frac{g+3}{2}\right\rfloor$. However, for the finite set of reduced graphs of genus $g$ this is not the case. For large enough $g$ the upper bound is not sharp. This can be seen by combining Lemma 5.4 with the inequality that bounds the number of vertices in the beginning of the proof of Lemma 5.5.

\section{Clifford's theorem}

We shall now prove the analog of Clifford's theorem. This is a theorem concerning vector spaces of cycles on a graph, and so may be formulated without the notion of squarefree modules. In algebraic geometry Clifford's theorem concerns linear systems on smooth projective curves. Graphs are in some sense singular, and in order for Clifford's theorem to hold we must have an extra assumption on our space of cycles. It must fulfill the criterion (to be defined) of being locally indecomposable at each vertex of the graph.

6.1. Definitions. We recall some notions from graph theory which will be necessary, and make several definitions needed in the statements and proofs of Clifford's theorem.

Let $G$ be a connected graph. A vertex $P$ of $G$ is called a cut point of $G$ if the removal of $P$ disconnects $G$. The maximal subgraphs of $G$ which have no cut points are called the blocks of $G$. A block consists either just of an edge, or it is a 2-connected graph. Note that each 
block of $G$ must contain at least one of the cut points of $G$. Those blocks which contain only one cut point will be called leaf blocks of $G$. The number of leaf blocks of $G$ is denoted by $l(G)$. If $Q$ is a cut point of $G$, then the removal of $Q$ and the incident edges disconnects $G$ into components $\hat{G}_{1}, \hat{G}_{2}, \ldots, \hat{G}_{r}$. Adding to $\hat{G}_{i}$ the edges in $G$ between $\hat{G}_{i}$ and $Q$, we get subgraphs $G_{1}, G_{2}, \ldots, G_{r}$ such that $G$ is their union and any pair intersects only in the cut point $Q$. These are the cut components of $G$ at the cut point $Q$. Note that $Q$ will not be a cut point of any of the $G_{i}$.

Let $G=(V, E)$ where $V$ are the vertices of $G$ and $E$ its edges. Let $C_{1}=k E$ be the one-chains of $G$. If $c$ is a chain, we define the support $\operatorname{supp} c$ to be the induced subgraph on the set of edges in $c$. The cycles $Z_{1}(G)$ is the kernel of the boundary map from the one-chains to the zero-chains

$$
C_{1}=k E \rightarrow k V=C_{0} .
$$

Lemma 6.1. Let $Q$ be a cutpoint of $G$ and $G_{1}, \ldots, G_{r}$ its cut components at $Q$. If $s$ is a cycle on $G$, then $s=s_{1}+s_{2}+\cdots+s_{r}$ where $s_{i}$ is a cycle on $G_{i}$. Hence there is an isomorphism

$$
Z_{1}(G) \cong \bigoplus_{i=1}^{r} Z_{1}\left(G_{i}\right) .
$$

Proof. Write $s=s_{1}+\cdots+s_{r}$ where $s_{i}$ is a chain in $G_{i}$. Then

$$
0=\partial(s)=\partial\left(s_{1}\right)+\cdots+\partial\left(s_{r}\right) .
$$

Now $\partial\left(s_{i}\right)$ is a zero chain in $G_{i}$. Every point $P$ involved in $\partial\left(s_{i}\right)$ must cancel against some point in $\partial\left(s_{j}\right)$ for some $j \neq i$. But since $G_{i}$ and $G_{j}$ only intersect in $Q$ we must have $\partial\left(s_{i}\right)=\alpha_{i} Q$ for a constant $\alpha_{i}$. Since $\partial^{2}\left(s_{i}\right)=0$, we get each $\alpha_{i}=0$, and so the $s_{i}$ are cycles in $G_{i}$.

A subspace $\Gamma \subseteq Z_{1}(G)$ of the cycles will be called a cycle system. The support supp $\Gamma$ is the union of the supports of all cycles in $\Gamma$. We shall assume the support of $\Gamma$ is $G$. Then each block of $G$ must be two-connected. In the case of the lemma above, let $\Gamma_{i}$ be the image of $\Gamma$ in $Z_{1}\left(G_{i}\right)$. We then get an injection

$$
\Gamma \hookrightarrow \bigoplus_{i=1}^{r} \Gamma_{i} .
$$

For each vertex $P \in V$, let $E_{P}$ be the set of edges incident to $P$ and let $C_{1 P}=k E_{P}$ be the vector space with these edges as basis. There is a natural map

$$
C_{1 P}=k E_{P} \rightarrow k P
$$

sending each edge $e \mapsto P$, and let $Z_{1 P}(G)$ be the kernel of this map, the local cycles at $P$. There is a natural map $C_{1} \rightarrow C_{1 P}$ and $Z_{1}(G)$ maps to $Z_{1 P}(G)$. 
If $\Gamma$ is a cycle system, we get for each vertex $P$ a map $\Gamma \rightarrow Z_{1 P}(G)$, and denote by $\Gamma_{P}$ the image of this. We define the degrees

$$
\begin{aligned}
d(\Gamma, P) & =\operatorname{dim}_{k} \Gamma_{P}-1 \\
d(\Gamma) & =\sum_{P \in V} d(\Gamma, P) .
\end{aligned}
$$

Now $\Gamma_{P} \subseteq Z_{1 P}(G)$ and this is again a subspace of $C_{1 P}=k E_{P}$. The latter is a vector space with a natural basis, the edges incident to $P$. Let us now for a moment consider this situation. Let $V$ be a vector space over the field $k$ with a basis $B=\left\{b_{1}, \ldots, b_{n}\right\}$. Let $\pi=B_{1} \cup B_{2} \cup \cdots \cup B_{r}$ be a partition of $B$ into nonempty parts. A subspace $U \subseteq V$ is decomposable with respect to $\pi$ if

$$
U \cong\left(U \cap k B_{1}\right) \oplus\left(U \cap k B_{2}\right) \oplus \cdots \oplus\left(U \cap k B_{r}\right) .
$$

We call $\Gamma$ locally decomposable at the vertex $P$ is there is a partition of $E_{P}$ such that $\Gamma_{P}$ is decomposable with respect to this partition; otherwise $\Gamma$ is locally indecomposable at the vertex $P$. We say that $\Gamma$ is locally indecomposable if it is locally indecomposable at all its vertices. We say $\Gamma \subseteq k E$ is decomposable if there is a partition of the set of edges such that $\Gamma$ is decomposable with respect to this partition; otherwise $\Gamma$ is indecomposable.

Let $P$ be a vertex where $\Gamma$ is decomposable. Let $E_{P}=E_{P 1} \cup E_{P 2}$ be a partition with respect to which $\Gamma_{P}$ is decomposable. We may now make a new graph $G^{\prime}$ by replacing the vertex $P$ by two vertices $P_{1}$ and $P_{2}$ and let the edges in $E_{P 1}$ be incident to $P_{1}$ and those in $E_{P 2}$ be incident to $P_{2}$. There is an exact sequence

$$
0 \rightarrow K_{P} \rightarrow \Gamma \rightarrow \Gamma_{P} \rightarrow 0
$$

where $K_{P}$ is the kernel. Note that

$$
\Gamma_{P}=\left(\Gamma_{P} \cap k E_{P_{1}}\right) \oplus\left(\Gamma_{P} \cap k E_{P_{2}}\right) .
$$

Define the kernels

$$
\begin{aligned}
& \Gamma_{1}=\operatorname{ker}\left(\Gamma \rightarrow\left(\Gamma_{P} \cap k E_{P_{2}}\right)\right) \\
& \Gamma_{2}=\operatorname{ker}\left(\Gamma \rightarrow\left(\Gamma_{P} \cap k E_{P_{1}}\right)\right) .
\end{aligned}
$$

For $i=1,2$ chose splittings of the surjections

$$
\Gamma_{i} \stackrel{\sigma_{i}}{\longleftarrow}\left(\Gamma_{P} \cap k E_{P i}\right) .
$$

We then have a decomposition

$$
\Gamma \cong K_{P} \oplus \operatorname{im} \sigma_{1} \oplus \operatorname{im} \sigma_{2} .
$$

There is an injection $Z_{1}\left(G^{\prime}\right) \hookrightarrow Z_{1}(G)$ and we see that the image of this map contains all the summands to the right of the above isomorphism. We then get a cycle system $\Gamma^{\prime}$ on $G^{\prime}$ mapping isomorphically onto $\Gamma$. This subspace $\Gamma^{\prime}$ does not depend on the choice of splittings. 
If $\Gamma^{\prime}$ is again locally decomposable at $P_{1}$ or $P_{2}$ we may continue the process. In the end we get a graph $\tilde{G}$ and a cycle system $\tilde{\Gamma}$ which is locally indecomposable at all vertices $Q$ of $\tilde{G}$ mapping to $P$. We call $(\tilde{G}, \tilde{\Gamma})$ the resolution of the pair $(G, \Gamma)$ at the vertex $P$.

6.2. Versions of Clifford's theorem. Clifford's theorem in algebraic geometry relates the dimension and degree of a linear system on a projective curve.

Theorem 6.2 (Clifford's theorem). Let D be an effective special divisor on a smooth projective curve $C$ over Speck. Let $\mathcal{L}(D)$ be the associated line bundle, and $\Gamma(\mathcal{L}(D))$ its global sections. Then $\operatorname{deg} D+2 \geq$ $2 \operatorname{dim}_{k} \Gamma(\mathcal{L}(D))$.

Here is our version for cycle systems on graphs.

Theorem 6.3 (Clifford's theorem). Let $G$ be a two-connected graph and $\Gamma$ a cycle system with support $G$. If $\Gamma$ is locally indecomposable, then

$$
d(\Gamma)+2 \geq 2 \operatorname{dim}_{k} \Gamma .
$$

Example 6.4. The following example shows that even if $\Gamma$ is indecomposable, it is necessary that $\Gamma$ be locally indecomposable for Clifford's theorem to hold. Let $G$ be the two-connected graph

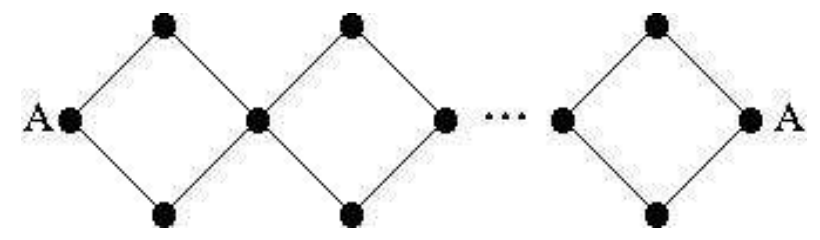

where the endpoints $A$ are identified. Let $c_{1}, c_{2}, \ldots, c_{r}$ be the fourcycles in the graph, and let $\Gamma$ be generated by the cycles

$$
c_{1}-c_{2}, c_{2}-c_{3}, \ldots, c_{r-1}-c_{r} \text {. }
$$

Then $\Gamma$ is indecomposable. But $\Gamma_{P}$ is locally decomposable at each vertex $P$. We have $d(\Gamma)=r$ and $\operatorname{dim}_{k} \Gamma=r-1$ so the inequality $d(\Gamma)+2 \geq 2 \operatorname{dim}_{k} \Gamma$ far from holds.

Corollary 6.5. Let $G$ be a two-connected graph where every vertex has degree at most three, and $\Gamma$ a cycle system with support $G$. Then

$$
d(\Gamma)+2 \geq 2 \operatorname{dim}_{k} \Gamma .
$$

Proof. In this case $\Gamma_{P}$ cannot be decomposable for any vertex $P$, so $\Gamma$ is locally indecomposable.

We will prove Theorem 6.3 through an inductive argument. It will then be necessary to have versions for the cases when the cycle systems may be locally decomposable.

If $G$ is a graph and $\Gamma$ a cycle system on $G$ we denote by $q(\Gamma)$ the number of vertices of $G$ at which $\Gamma$ is locally decomposable. When $G$ 
has connectivity one a leaf block of $G$ will be called regular if all its vertices are locally indecomposable, except perhaps the vertex to which it is attached to $G$ (the cutpoint). Let $l_{\text {reg }}(\Gamma)$ be the number of regular leaf blocks of $G$.

Proposition 6.6. Let $G$ be a graph of connectivity one, and $\Gamma$ a cycle system with support $G$. Then

$$
d(\Gamma)+q(\Gamma)+l_{\text {reg }}(\Gamma) \geq 2 \operatorname{dim}_{k} \Gamma .
$$

Proposition 6.7. Let $G$ be a two-connected graph, and $\Gamma$ a cycle system with support $G$. Suppose the number of locally decomposable vertices is $q(\Gamma) \geq 1$. Then

$$
d(\Gamma)+q(\Gamma) \geq 2 \operatorname{dim}_{k} \Gamma .
$$

In order to formulate Clifford's theorem for modules, we define a $k[G]$-module $M$ to be indecomposable at a vertex $i$ if the submodule $M_{\geq i}$ of $M$, consisting of the multigraded pieces of $M$ indexed by multidegrees $\geq i$, is an indecomposable module. The module $M$ is locally indecomposable if it is indecomposable at each vertex $i$ of $G$. Formulated in terms of modules we get.

Theorem 6.8 (Clifford's theorem). Let $G$ be a two-connected graph and $M$ a special effective $k[G]$-module. If $M$ is locally indecomposable, then

$$
\operatorname{deg}(M)+2 \geq 2 l(M) .
$$

Proof. Let $N$ be the submodule of $M$ generated by the sections $M_{\emptyset}$. Then $N$ is also special and effective. Let $q$ be the number of vertices at which $N$ is locally decomposable. Then $\operatorname{deg}(M) \geq \operatorname{deg}(N)+q$. If $q=0$ the statement follows by Theorem 6.3. If $q \geq 1$ we have by Proposition 6.7

$$
\operatorname{deg}(M) \geq \operatorname{deg}(N)+q \geq 2 l(N)=2 l(M),
$$

which implies the inequality of the theorem.

In the same way as we got Corollary 6.5 we get the following.

Corollary 6.9. Let $G$ be a two-connected graph where every vertex has degree at most three, and $M$ a special effective $k[G]$-module. Then

$$
\operatorname{deg}(M)+2 \geq 2 l(M) .
$$

6.3. The proofs. We will prove Theorem 6.3 and Propositions 6.6 and 6.7 by induction on the number of locally decomposable vertices and the number of edges of $G$. The argument will be simultaneous induction in the sense that when we prove one of the statements we assume that all three statements hold if the number of edges is strictly smaller or the number of edges is the same, but the number of locally decomposable vertices is strictly smaller. 
Proof of Proposition 6.6. Let $Q$ be a cut point of $G$. If $F$ is a cut component of $G$ at $Q$, then $\Gamma$ induces a cycle system $\Phi$ on $F$. For all points $P$ in $F$ distinct from $Q$ we will have $\Phi_{P}=\Gamma_{P}$. Note that the point $Q$ on $F$ will not be a cut point of $F$.

If $F$ has connectivity one, either $Q$ will be in a nonleaf block of $F$, denote the set of such $F$ 's by $N L^{1}$, or $Q$ will be in a leaf block of $F$. This leaf block may either be regular, denote the set of such $F$ 's by $L_{\text {reg }}^{1}$, or non-regular, denote the set of such $F^{\prime}$ 's by $L_{n r e g}^{1}$. Let $C^{1}$ be the union of these three sets, the set of $F$ 's of connectivity one.

Let now $F$ be two-connected. Either $\Phi$ is locally indecomposable, denote the set of such $F$ 's by $B_{\text {reg }}^{2}$, or the only locally decomposable vertex for $\Phi$ is $Q$, denote the set of such $F$ 's by $B_{Q}^{2}$, or $\Phi$ contains a vertex distinct from $Q$ which is locally decomposable, denote the set of such $F^{\prime}$ 's by $B_{n r e g}^{2}$. Let $C^{2}$ be the union of these three sets, the set of two-connected F's. Finally let $C=C^{1} \cup C^{2}$ be the set of all the components $F$.

By induction, when $F$ has connectivity one, we have

$$
d(\Phi)+q(\Phi)+l_{\text {reg }}(\Phi) \geq 2 \operatorname{dim}_{k} \Phi,
$$

and when $F$ is two-connected we have

$$
\begin{aligned}
d(\Phi)+2 & \geq 2 \operatorname{dim}_{k} \Phi, \quad(F, \Phi) \in B_{r e g}^{2} \\
d(\Phi)+q(\Phi) & \geq 2 \operatorname{dim}_{k} \Phi, \quad(F, \Phi) \in B_{Q}^{2} \cup B_{n r e g}^{2}
\end{aligned}
$$

If $F$ has connectivity one and is in $N L^{1}$ or $L_{n r e g}^{1}$, the regular leaf blocks of $F$ will still be regular leaf blocks in $G$, but when $F$ is in $L_{\text {reg }}^{1}$, the attaching regular leaf block will cease to be a leaf block. The two-connected $F$ 's which are in $B_{\text {reg }}^{2}$ and $B_{Q}^{2}$ will become regular leaf blocks of $G$, but not the ones in $B_{n r e g}^{2}$. We therefore have

$$
\begin{aligned}
l_{\text {reg }}(\Gamma) & =\sum_{(F, \Phi) \in N L^{1}} l_{\text {reg }}(\Phi)+\sum_{(F, \Phi) \in L_{\text {rreg }}^{1}} l_{\text {reg }}(\Phi) \\
& +\sum_{(F, \Phi) \in L_{r e g}^{1}}\left(l_{\text {reg }}(\Phi)-1\right)+\left|B_{r e g}^{2}\right|+\left|B_{Q}^{2}\right| \\
& =\sum_{(F, \Phi) \in C^{1}} l_{\text {reg }}(\Phi)+\left|B_{\text {reg }}^{2}\right|+\left|B_{Q}^{2}\right|-\left|L_{\text {reg }}^{1}\right| .
\end{aligned}
$$

Concerning the locally decomposable vertices with respect to $\Phi$, when $F$ is in $N L^{1}$ or $L_{n r e g}^{1}$ we may lose a decomposable vertex when going from $\Phi$ to $\Gamma$, since $Q$ may be changing its status from locally decomposable to locally indecomposable. Similarly for $F$ in $B_{Q}^{2} \cup B_{\text {nreg }}^{2}$. 
Hence

$$
\begin{aligned}
q(\Gamma) & \geq \sum_{(F, \Phi) \in N L^{1} \cup L_{n r e g}^{1}}(q(\Phi)-1)+\sum_{(F, \Phi) \in L_{r e g}^{1}} q(\Phi) \\
& +\sum_{(F, \Phi) \in B_{Q}^{2} \cup B_{n r e g}^{2}}(q(\Phi)-1)+\epsilon \\
& =\sum_{(F, \Phi) \in C} q(\Phi)-\left|N L^{1}\right|-\left|L_{n r e g}^{1}\right|-\left|B_{Q}^{2}\right|-\left|B_{n r e g}^{2}\right|+\epsilon,
\end{aligned}
$$

where $\epsilon=1$ if $\Gamma$ is locally decomposable at $Q$, and $\epsilon=0$ if $\Gamma$ is locally indecomposable at $Q$.

By (3) there is a commutative diagram

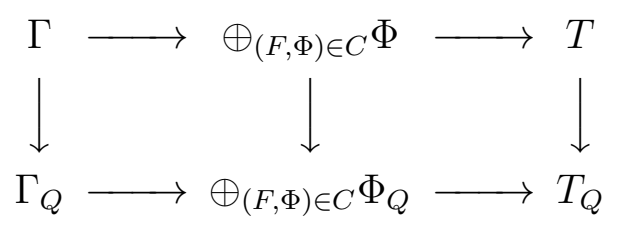

where $T$ and $T_{Q}$ are the cokernels of the injections on the left. Since each $\Phi \rightarrow \Phi_{Q}$ is surjective, there is a surjection $T \rightarrow T_{Q}$.

From this we obtain

$$
\begin{aligned}
d(\Gamma, Q)+1 & =\sum_{(F, \Phi) \in C}(d(\Phi, Q)+1)-\operatorname{dim} T_{Q} \\
d(\Gamma, Q) & =\sum_{(F, \Phi) \in C} d(\Phi, Q)+|C|-1-\operatorname{dim}_{k} T_{Q}
\end{aligned}
$$

At all points $P$ of $F$ distinct from $Q$ we have $d(\Gamma, P)=d(\Phi, P)$. Therefore

$$
d(\Gamma)=\sum_{(F, \Phi) \in C} d(\Phi)+|C|-1-\operatorname{dim}_{k} T_{Q}
$$

Now we add the equations obtained by induction (5), (6), and (77) and get

$$
\sum_{(F, \Phi) \in C} d(\Phi)+\sum_{(F, \Phi) \in C} q(\Phi)+2\left|B_{r e g}^{2}\right|+\sum_{(F, \Phi) \in C^{1}} l_{r e g}(\Phi) \geq 2 \sum_{(F, \Phi) \in C} \operatorname{dim}_{k} \Phi
$$

Equations (13), (9), and (8) give expressions for $d(\Gamma), q(\Gamma)$ and $l_{\text {reg }}(\Gamma)$ respectively. Inserting these in the above equation and taking into account the upper row in the commutative diagram (10), we obtain

$$
\begin{aligned}
& d(\Gamma)-|C|+1+\operatorname{dim}_{k} T_{Q} \\
+ & q(\Gamma)+\left|N L^{1}\right|+\left|L_{\text {nreg }}^{1}\right|+\left|B_{Q}^{2}\right|+\left|B_{\text {nreg }}^{2}\right|-\epsilon \\
+ & 2\left|B_{\text {reg }}^{2}\right|+l_{\text {reg }}(\Gamma)+\left|L_{\text {reg }}^{1}\right|-\left|B_{Q}^{2}\right|-\left|B_{\text {reg }}^{2}\right| \\
\geq & 2 \operatorname{dim}_{k} \Gamma+2 \operatorname{dim}_{k} T .
\end{aligned}
$$


The terms containing the cardinalities of sets of cut components are

$$
-|C|+\left|N L^{1}\right|+\left|L_{\text {nreg }}^{1}\right|+\left|L_{\text {reg }}^{1}\right|+\left|B_{\text {reg }}^{2}\right|+\left|B_{\text {nreg }}^{2}\right| \leq 0 .
$$

Hence we get

$$
d(\Gamma)+l_{\text {reg }}(\Gamma)+q(\Gamma) \geq 2 \operatorname{dim}_{k} \Gamma+\left(2 \operatorname{dim}_{k} T-\operatorname{dim} T_{Q}+\epsilon-1\right) .
$$

If $\operatorname{dim} T \geq 1$, the expession in the paranthesis on the right is $\geq 0$ since $\operatorname{dim}_{k} T \geq \operatorname{dim}_{k} T_{Q}$. If $\operatorname{dim}_{k} T=0$ then $\epsilon=1$ and so the paranthesis is also $\geq 0$. Hence we obtain

$$
d(\Gamma)+l_{\text {reg }}(\Gamma)+q(\Gamma) \geq 2 \operatorname{dim}_{k} \Gamma .
$$

Proof of Proposition 6.7. Let $P$ be a locally decomposable vertex on $G$ for the cycle system $\Gamma$ and let $(\tilde{G}, \tilde{\Gamma})$ be the resolution of $(G, \Gamma)$ at the vertex $P$. Since $G$ is two-connected, $\tilde{G}$ will be connected.

If $\tilde{G}$ is two-connected we will have $q(\tilde{\Gamma})=q(\Gamma)-1$ and $d(\Gamma) \geq$ $d(\tilde{\Gamma})+1$. If $q(\tilde{\Gamma}) \geq 1$ we have by induction

$$
d(\tilde{\Gamma})+q(\tilde{\Gamma}) \geq 2 \operatorname{dim}_{k} \tilde{\Gamma},
$$

and so we obtain

$$
\begin{aligned}
& d(\Gamma)+q(\Gamma) \geq d(\tilde{\Gamma})+q(\tilde{\Gamma})+2 \\
\geq \quad & 2 \operatorname{dim}_{k} \tilde{\Gamma}+2=2 \operatorname{dim}_{k} \Gamma+2 \geq 2 \operatorname{dim}_{k} \Gamma .
\end{aligned}
$$

If $q(\tilde{\Gamma})=0$ we have by induction

$$
d(\tilde{\Gamma})+2 \geq 2 \operatorname{dim} \tilde{\Gamma}
$$

SO

$$
d(\Gamma)+q(\Gamma)=d(\Gamma)+1 \geq d(\tilde{\Gamma})+2 \geq 2 \operatorname{dim}_{k} \Gamma .
$$

Suppose now $\tilde{G}$ has connectivity one. Then

$$
d(\tilde{\Gamma})+q(\tilde{\Gamma})+l_{\text {reg }}(\tilde{\Gamma}) \geq 2 \operatorname{dim}_{k} \tilde{\Gamma} .
$$

Let $D$ be the set of vertices in $\tilde{G}$ which map to $P$ in $G$. Then each leaf block of $\tilde{G}$ must contain an element of $D$ which is not the vertex at which the leaf block is attached to $\tilde{G}$ (the cut point). Otherwise the leaf block maps to a leaf block of $G$, which is impossible since $G$ is two-connected. Thus $|D| \geq l(\tilde{G})$.

Now we have $d(\Gamma)=d(\tilde{\Gamma})+|D|-1$. Also $q(\Gamma)=q(\tilde{\Gamma})+1$. We therefore get

$$
\begin{aligned}
d(\Gamma)+q(\Gamma)=d(\tilde{\Gamma})+q(\tilde{\Gamma})+|D| & \geq d(\tilde{\Gamma})+q(\tilde{\Gamma})+l(\tilde{G}) \\
& \geq d(\tilde{\Gamma})+q(\tilde{\Gamma})+l_{\text {reg }}(\tilde{\Gamma}) \\
& \geq 2 \operatorname{dim}_{k} \tilde{\Gamma}=2 \operatorname{dim}_{k} \Gamma .
\end{aligned}
$$

Finally we are able to prove Clifford's theorem. 
Proof of Theorem 6.3. Choose a cycle $s$ in $\Gamma$, and an edge $e$ on $s$. Let $\Gamma_{0}$ be the subspace of $\Gamma$ consisting of cycles not containing the edge $e$. Then $\Gamma=\Gamma_{0} \oplus k s$. Let $G_{0}$ be the support of $\Gamma_{0}$, so $G_{0}$ has less edges than $G$. On each connected component $F$ of $G_{0}$ the cycle system $\Gamma_{0}$ induces a cycle system $\Phi$. Denote by $C^{1}$ the set of components of $G_{0}$ of connectivity one, and by $C^{2}$ the set of components of $G_{0}$ which are two-connected. We may decompose $C^{2}=C_{\text {reg }}^{2} \cup C_{\text {nreg }}^{2}$ where $C_{\text {reg }}^{2}$ are the components $F$ of $G_{0}$ where the induced cycle system $\Phi$ is locally indecomposable, and $C_{n r e g}^{2}$ are those where $\Phi$ has some vertex which is locally decomposable.

Now we have

$$
\begin{aligned}
d(\Phi)+l_{\text {reg }}(\Phi)+q(\Phi) & \geq 2 \operatorname{dim}_{k} \Phi, \quad(F, \Phi) \in C^{1} \\
d(\Phi)+2 & \geq 2 \operatorname{dim}_{k} \Phi, \quad(F, \Phi) \in C_{r e g}^{2} \\
d(\Phi)+q(\Phi) & \geq 2 \operatorname{dim}_{k} \Phi, \quad(F, \Phi) \in C_{\text {rreg }}^{2}
\end{aligned}
$$

Let $T$ be the set of vertices $P$ of $G_{0}$ where $\operatorname{dim}_{k} \Gamma_{P}>\operatorname{dim}_{k} \Gamma_{0 P}$. We may decompose $T=T^{1} \cup T^{2}$ where $T^{1}$ are the vertices in $T$ contained in components of connectivity one and $T^{2}$ the vertices which are in two-connected components. Furthermore $T^{1}=T_{\text {reg }}^{1} \cup T_{n r e g}^{1}$ where $T_{r e g}^{1}$ are the points which are in regular leaf blocks, and are not cutpoints in the component they belong to, and $T_{n r e g}^{1}$ are the rest of the points in $T^{1}$. Also $T^{2}=T_{\text {reg }}^{2} \cup T_{n r e g}^{2}$ where $T_{\text {reg }}^{2}$ are the points of $T$ which are on components in $C_{\text {reg }}^{2}$ and $T_{n r e g}^{2}$ are the points of $T$ which are on components of $C_{n r e g}^{2}$.

As we now explain the following three inequalities hold:

$$
\begin{aligned}
\left|T_{r e g}^{1}\right| & \geq \sum_{(F, \Phi) \in C^{1}} l_{r e g}(\Phi), \\
\left|T_{\text {nreg }}^{1} \cup T_{\text {nreg }}^{2}\right| & \geq \sum_{(F, \Phi) \in C} q(\Phi), \\
\left|T_{r e g}^{2}\right| & \geq 2\left|C_{r e g}^{2}\right| .
\end{aligned}
$$

The first inequality is because a regular leaf block $L$ of $F$ must contain a vertex of $T^{1}$ which is not the vertex at which the leaf block is attached to the rest of $F$ (the cut point). Otherwise the cycle $s$ can only enter and leave $L$ at the cut point, and so $L$ will be a leaf block of $G_{0} \cup \operatorname{supp} s=G$, contrary to $G$ being two-connected.

The second inequality is because $\Gamma_{P}$ is indecomposable at every point $P$. So at all points $P$ at which $\Phi$ is decomposable we must have $\operatorname{dim}_{k} \Gamma_{P}>\operatorname{dim}_{k} \Gamma_{0 P}=\operatorname{dim}_{k} \Phi_{P}$.

The third inequality is because the cycle $s$ must enter a two-connected component $F$ in at least two points in order to avoid $F$ becoming a leaf block in $G$. 
Adding the equations (14) obtained by induction we get:

$\sum_{(F, \Phi) \in C} d(\Phi)+\sum_{(F, \Phi) \in C^{1}} l_{\text {reg }}(\Phi)+\sum_{(F, \Phi) \in C} q(\Phi)+2\left|C_{r e g}^{2}\right| \geq 2 \sum_{(F, \Phi) \in C} \operatorname{dim}_{k} \Phi$

Now

$$
d(\Gamma)=\sum_{(F, \Phi) \in C} d(\Phi)+|T|
$$

Using this and the inequalities (15) we obtain

$$
\begin{aligned}
& d(\Gamma)-|T|+\left|T_{\text {reg }}^{1}\right|+\left|T_{\text {nreg }}^{1}\right|+\left|T_{\text {nreg }}^{2}\right|+\left|T_{\text {reg }}^{2}\right| \\
\geq & 2 \sum_{(F, \Phi) \in C} \operatorname{dim}_{k} \Phi \geq 2 \operatorname{dim} \Gamma_{0} .
\end{aligned}
$$

Since $T$ is the union of $T_{r e g}^{1}, T_{n r e g}^{1}, T_{r e g}^{2}$ and $T_{n r e g}^{2}$ we obtain

$$
d(\Gamma) \geq 2 \operatorname{dim}_{k} \Gamma_{0}
$$

and since $\operatorname{dim}_{k} \Gamma_{0}=\operatorname{dim}_{k} \Gamma-1$ we get

$$
d(\Gamma)+2 \geq 2 \operatorname{dim}_{k} \Gamma
$$

Remark 6.10. An interesting topic for further work was pointed out by F.O. Schreyer. When the graph is trivalent, the multiplication in the canonical module gives rise to a line configuration in $\mathbb{P}^{g-1}$ in the following way. The projectification of the surjective multiplication maps $\widetilde{H}_{d-1}(G) \cong\left(\omega_{k[G]}\right)_{0} \stackrel{x_{i}}{\rightarrow}\left(\omega_{k[G]}\right)_{\mathbf{e}_{i}} \cong \widetilde{H}_{d-1}(G ; G-\{i\})$ is a line in $\mathbb{P}^{g-1}$. The line configuration of the union of all these lines is a graph curve of arithmetic genus $g$ which is canonically embedded. Furthermore, a $g_{d}^{1}$ for the graph gives a projection of this curve to a $\mathbb{P}^{1}$ of degree $d$. It is therefore natural to ask if the gonality of the graph and this curve is the same. Similar curves can also be made from more general 2-connected graphs, by choosing appropriate rational curves in the corresponding union of projective spaces. One interesting question is if there is a connection between the gonality (or an appropriately defined Clifford index) of the graph and the minimal free resolution of this canonical curve as in Green's conjecture for a canonical curve, which says that you can read the Clifford index of a curve of the graded betti numbers of the minimal free resolution. In many examples we made, this seems to be the case, but it seems very difficult to show this in general. This is first of all because the ideal of the canonical curve is not multigraded, and there is not a combinatorial way to describe the minimal free resolution. Secondly, the Clifford index of a graph is also not easily computed. 


\section{REFERENCES}

[1] K. Baclawski. Canonical modules of partially ordered sets. J. Algebra, 83(1):1$5,1983$.

[2] M. Baker and S. Norine. Riemann-Roch and Abel-Jacobi theory on a finite graph. Advances in Math., 215(2):766-788, 2007.

[3] D. Bayer and D. Eisenbud. Graph curves. Advances in Math., 86:1-40, 1991.

[4] W. Bruns and J. Herzog. Cohen-Macaulay rings. Cambridge University Press, 1998.

[5] P.J. Cameron. Combinatorics: Topics, Techniques, Algorithms. Cambridge university press, 1994.

[6] D. Eisenbud. Commutative Algebra with a View Toward Algebraic Geometry, volume 150 of Graduate Texts in Mathematics. Springer-Verlag, 1994.

[7] E. Miller. The Alexander duality functors and local duality with monomial support. J. Algebra, 231(1):180-234, 2000.

[8] E. Miller and B. Sturmfels. Combinatorial Commutative Algebra, volume 227 of GTM. Springer, 2000.

[9] R.P. Stanley. Combinatorics and commutative algebra. Birkhauser, 1996.

[10] K. Yanagawa. Alexander duality for Stanley-Reisner rings and square-free $\mathbf{N}^{n}$ graded modules. J. Algebra, 225(2):630-635, 2000.

[11] K. Yanagawa. Derived category of squarefree modules and local cohomology with monomial ideal support. J. Math. Soc. Japan, 56(1):289-308, 2004.

Matematisk Institutt, Johs. Brunsgt. 12, 5008 Bergen

E-mail address: gunnar@mi.uib.no

Matematisk Institutt, Johs. Brunsgt. 12, 5008 Bergen

E-mail address: henning.lohne@math.uib.no 